Pure and Applied Mathematics Quarterly

Volume 3, Number 2

(Special Issue: In honor of

Leon Simon, Part 1 of 2)

$569-594,2007$

\title{
Existence and Regularity of Stable Branched Minimal Hypersurfaces
}

\author{
Neshan Wickramasekera
}

This is primarily an expository article focused upon the recent work of the author on the local structure of stable branched minimal hypersurfaces and the joint work of L. Simon and the author on the existence of stable branched minimal immersions in a Euclidean space of arbitrary dimension $\geq 3$. In Section 1, we discuss the main regularity and compactness theorems, established in [Wic-1] and [Wic-2], for immersed stable minimal hypersurfaces. Section 2 contains a description of a method, established in $[\mathrm{SW}]$, which shows the existence in Euclidean space of a rich class of stable minimal hypersurfaces with branch point singularities.

A secondary objective here concerns a rather technical point; that is, we here observe, in Section 3, an improvement of the asymptotic analysis of the 2-valued harmonic functions (which arise as "blow-ups" of sequences of stable minimal hypersurfaces weakly converging to multiplicity 2 hyperplanes) carried out in [Wic-1]. Note that the graphs of these functions serve as approximations to stable minimal hypersurfaces whenever the hypersurfaces are weakly close to hyperplanes, and a quantitative version of this approximation was needed in the proof of the main excess improvement lemma of [Wic-1] (Lemma 1.8 below), which in turn was the key ingredient of the proof of the main regularity result of [Wic-1] (Theorem 1.3 below). The viewpoint offered here leads directly to a more complete version of the main a priori regularity estimate for the 2-valued harmonic functions (Theorem 5.1 of [Wic-1], reproduced as Theorem 1.9 below) and, consequently, to a simpler version of the excess improvement lemma for stable minimal hypersurfaces. We here show that these 2-valued harmonic functions in fact satisfy an a priori estimate which is the direct analogue of the corresponding

Received August 23, 2005.

Partly supported by NSF grant DMS-0406447 at UCSD 
(well-known) estimate for single valued harmonic functions. As a direct consequence, we obtain a simplification of the excess improvement lemma for stable minimal hypersurfaces which in particular eliminates the need, in order to obtain excess improvement, to allow "multiple scales", as was done in the original version of the lemma in [Wic-1]. Indeed, in this simpler version, the improvement of height excess of the minimal submanifold is shown to occur always at a single fixed smaller scale, much like in the context of all other well known regularity theorems of the kind established in [Wic-1].

\section{REGULARITY AND COMPACTNESS OF STABLE MINIMAL HYPERSURFACES}

In [SS81], R. Schoen and L. Simon developed a partial regularity theory for $n$-dimensional stable minimal hyperpsurfaces $M$ of an open ball of a Euclidean space (or any given Riemannian manifold) assuming that the (regular parts of the) hypersurfaces are embedded and their singularities have locally finite $(n-2)$ dimensional Hausdorff measure. The main result in [SS81] (Theorem 2 there) is the following compactness theorem.

Theorem 1.1. (Schoen-Simon) Suppose $M_{k}$ is a sequence of orientable embedded, stable minimal hypersurfaces of $B_{2}^{n+1}(0)$ with $0 \in \bar{M}_{k}, \mathcal{H}^{n-2}\left(\operatorname{sing} M_{k}\right)<\infty$ for $k=1,2,3, \ldots$ and

$$
\limsup _{k \rightarrow \infty} \mathcal{H}^{n}\left(M_{k}\right)<\infty
$$

Then there exist a stationary integral varifold $V$ of $B_{2}^{n+1}(0)$ and a closed subset $S$ of spt $\|V\| \cap B_{1}^{n+1}(0)$ with $S=\emptyset$ if $2 \leq n \leq 6, S$ discrete if $n=7$ and $\mathcal{H}^{n-7+\gamma}(S)=0$ for every $\gamma>0$ if $n \geq 8$ such that, after passing to a subsequence of $\{k\}$ which we again denote $\{k\}, M_{k} \rightarrow V$ as integral varifolds in $B_{2}^{n+1}(0)$ and (spt $\|V\| \backslash S) \cap B_{1}^{n+1}(0)$ is an orientable, embedded, smooth stable minimal hypersurface of $B_{1}^{n+1}(0)$.

Here and subsequently, $B_{\rho}^{n+1}(X)$ denotes the open ball of $\mathbf{R}^{n+1}$ with radius $\rho$ and center $X$ and $\mathcal{H}^{m}$ denotes the $m$-dimensional Hausdorff measure in $\mathbf{R}^{n+1}$; $\|V\|$ denotes the weight measure associsted with the varifold $V$, and spt $\|V\|$ the support of $\|V\|$. We shall identify the hyperplane $\left\{x^{n+1}=0\right\}$ of $\mathbf{R}^{n+1}$ with $\mathbf{R}^{n}$ and denote by $B_{\rho}(X)$ the open ball of $\mathbf{R}^{n}$ with radius $\rho$ and center $X$. 
The main ingredient of the proof of the above theorem is the following small excess regularity theorem (Theorem 1 of [SS81]).

Theorem 1.2. (Schoen-Simon) Let $\Lambda \in(1, \infty)$. There exists a number $\epsilon=$ $\epsilon(n, \Lambda) \in(0,1)$ such that the following holds: Let $M \subset B_{2}^{n+1}(0)$ be an embedded, stable minimal hypersurface with $0 \in \bar{M}, \mathcal{H}^{n-2}(\operatorname{sing} M)<\infty$ and $\frac{\mathcal{H}^{n}(M)}{\omega_{n} 2^{n}} \leq \Lambda$. If $E^{2} \equiv \int_{M \cap\left(B_{1}(0) \times \mathbf{R}\right)}\left|x^{n+1}\right|^{2} \leq \epsilon$, then $\bar{M} \cap\left(B_{1 / 2}(0) \times \mathbf{R}\right)=\cup_{i=1}^{k}$ graph $u_{k}$ where $u_{i}: B_{1 / 2}(0) \rightarrow \mathbf{R}$ are $C^{2}$ functions satisfying $u_{1}<u_{2}<\ldots<u_{k}$ and $\left\|u_{i}\right\|_{C^{2}\left(B_{1 / 2}(0)\right)} \leq C E$. Here $\omega_{n}$ denotes the volume of the unit ball in $\mathbf{R}^{n}$ and $C=C(n) \in(0, \infty)$.

Remarks: In the following remarks, $M$ refers to a hypersurface as in Theorem 1.2. Remarks (1), (2) and (3) taken with $M_{k}, k=1,2,3, \ldots$, in place of $M$ also apply to Theorem 1.1.

(1) $M$ is assumed to be equal to the regular part of the hypersurface. i.e. $M$ has no removable singularities. Thus, with $B=B_{2}^{n+1}(0), M$ is equal to the set of points $X \in \bar{M} \cap B$ such that for some $\sigma>0$, $\bar{M} \cap \overline{B_{\sigma}^{n+1}(X)}$ is a connected, compact, embedded $n$-dimensional submanifold of $B_{\sigma}^{n+1}(X)$ with its boundary contained in $\partial B_{\sigma}^{n+1}(X)$. The interior singular set $\operatorname{sing} M$ of $M$ is then defined by

$$
\operatorname{sing} M=(\bar{M} \backslash M) \cap B .
$$

(2) Minimality (or stationarity) of $M$ in $B$ means that $M$ has zero first variation of volume with respect to deformations by $C^{1}$ vector fields of the ambient space $B$ with compact support. This is equivalent to the condition (see e.g. [Sim83], Section 9) that

$$
\int_{M} \operatorname{div} X d \mathcal{H}^{n}=0 \quad \text { for each } \quad X \in C_{c}^{1}\left(B ; \mathbf{R}^{n+1}\right) .
$$

(3) Let $M$ be stationary in $B$. Then $M$ is stable if $M$ has non-negative second variation of volume with respect to deformations as in (2). Since $M$ has codimension 1 , and $\mathcal{H}^{n-2}(\operatorname{sing} M)<\infty$, stability of $M$ is equivalent to ([Sim83], Section 9)

$$
\int_{M}|A|^{2} \zeta^{2} \leq \int_{M}|\nabla \zeta|^{2} \quad \text { for each } \quad \zeta \in C_{c}^{1}(M)
$$


where $A$ denotes the second fundamental form of $M$ and $|A|$ the length of $A$.

(4) In [SS81], Theorem 1.2 was actually proved under the assumption that $\mathcal{H}^{n-2}(\operatorname{sing} M)=0$ (and, consequently, Theorem 1.1 under the assumption that $\mathcal{H}^{n-2}\left(\operatorname{sing} M_{k}\right)=0$ for each $\left.k=1,2,3, \ldots\right)$. This hypothesis can however be weakened to $\mathcal{H}^{n-2}(\operatorname{sing} M)<\infty$ without changing the conclusions, so the theorems indeed hold as stated above. (See [Wic-1], Section 3 for a justification of this claim.) In contrast, if we do not assume embeddedness of $M$, although a similar theorem holds (at least with a specific mass bound, see Theorems 1.3 and 1.4 below), the conclusions depend on whether we assume that $\mathcal{H}^{n-2}(\operatorname{sing} M)=0$ or merely $\mathcal{H}^{n-2}(\operatorname{sing} M)<\infty$. In the former case, we have decomposition into (possibly intersecting) regular graphs, but in the latter case there may be a singular (branching) set present giving a 2-valued graph as the best possible conclusion. See also Theorem 2.3 below.

Schoen-Simon theory is a "multiplicity 1" theory. Indeed, by a result of $\mathrm{T}$. Ilmanen [Ilm96] (which uses Theorem 1.1), if $M$ is an embedded stable minimal hypersurface of an open ball $B \subset \mathbf{R}^{n+1}$ satisfying $\mathcal{H}^{n-2}(\operatorname{sing} M \cap B)<\infty$, then any tangent cone to $M$ at a point $Z \in \bar{M} \cap B$ is a multiplicity 1 cone. i.e. multiple "sheets" cannot come together when passing to the weak limit of a sequence of geometric rescalings of $M$ at $Z$.

Of course stable minimal hypersurfaces need not always be embedded as is demonstrated by the simple example of the union of a pair of transversely intersecting hyperplanes. In this example, we consider the points where the hyperplanes intersect as regular points of the union, as we should. Thus, it makes sense to generalize the above definition of interior regular point of a hypersurface and define singular point as follows:

\section{Definitions:}

(1) Let $B$ be an open ball in $\mathbf{R}^{n+1}$ and $M \subset B$ an immersed hypersurface. Thus, for each $X \in M$, there exists $\sigma>0$ such that $M \cap \overline{B_{\sigma}^{n+1}(X)}$ is the union of a finite number of smooth, embedded, compact, connected, $n$-dimensional submanifolds of $\overline{B_{\sigma}^{n+1}(X)}$ with boundary contained in $\partial B_{\sigma}^{n+1}(X)$.

(2) Let $M, B$ be as in (1). A point $X \in \bar{M} \cap B$ is an interior regular point of $M$ if there exists $\sigma>0$ such that $\bar{M} \cap \overline{B_{\sigma}^{n+1}(X)}$ is equal to the union of a 
finite number of smooth, embedded, compact, connected, $n$-dimensional submanifolds of $\overline{B_{\sigma}^{n+1}(X)}$ each with its boundary contained in $\partial B_{\sigma}^{n+1}(X)$. The interior regular part reg $M$ of $M$ is the collection of all of its interior regular points. Thus $M \subseteq \operatorname{reg} M$. By redefining $M$ if necessary, we shall assume, as we did in Theorems 1.1 and 1.2 (see Remark 1 above), that $M=\operatorname{reg} M$. Subject to this assumption, the interior singular set $\operatorname{sing} M$ of $M$ is defined by $\operatorname{sing} M=(\bar{M} \backslash M) \cap B$. (Thus $M$ has no removable singularities.)

With these definitions, we seek a regularity theory for the class of immersed hypersurfaces $M$ of an open ball $B \subset \mathbf{R}^{n+1}$ satisfying $\mathcal{H}^{n}(M)<\infty$ which are stationary and stable points of the volume functional on hypersurfaces of $B$. Here stationarity and stability are defined exactly as described in Remarks 2 and 3 above, and amount to the conditions $(S 1)$ and $(S 2)$. In order to be able to make good use of the stability assumption, one has to impose a priori some restriction on the size of the singular sets of the hypersurfaces. A natural hypothesis, which is weak enough to allow branch point singularities and strong enough to give good $L^{2}$-control of the (extrinsic) curvature (via stability) is that

$$
\mathcal{H}^{n-2}(\operatorname{sing} M)<\infty
$$

One of the main goals of such a theory is to understand the nature of $\sin g M$, and at the heart of the theory are appropriate small excess regularity theorems analogous to Theorem 1.2. (See Theorems 1.3 and 1.5 below.)

What is the main additional difficulty arising from dropping the embeddedness hypothesis? The short answer to this is higher multiplicity. It is possible, in the absence of the embeddedness assumption, for a tangent cone to $M$ at a singular point $Z$ to have multiplicity $>1$. This possibility indeed does occur. See the construction described in Section 2 below. From the viewpoint of the complexity of tangent cones, the simplest such singularities are the ones where the tangent cones are sums of (varifolds associated with) hyperplanes with multiplicity $\geq 1$. (Recall that by Allard's regularity theorem ([All72], [Sim83]), if a tangent cone at a point is a single, multiplicity 1 hyperplane, then that point is a regular point of the hypersurface.) We shall call a point $Z \in \operatorname{sing} M$ a branch point of $M$ if some tangent cone to $M$ at $Z$ is a sum of hyperplanes $P$ with multiplicity $m_{P} \geq 1$. Two basic questions related to the study of branch points (and for any other kind of singularities for that matter) are: (1) whether the tangent cones at the branch points are unique and (2) what the asymptotic behavior of the hypersurface is 
near its branch points. These are highly non-trivial questions intimately connected with each other - ones that remain open except in the simplest case when a tangent cone is a single hyperplane $P$ with $m_{P}=2$. This case is addressed in [Wic-1]; see Theorem 1.3 below. Notice that with $B=B_{2}^{n+1}(0)$, all tangent cones at branch points of $M$ in a smaller ball will be multiplicity 2 hyperplanes if we make the assumption (as we do in [Wic-1], [Wic-2] and [Wic04]) that for a given, fixed $\delta \in(0,1)$,

$$
\frac{\mathcal{H}^{n}(M)}{\omega_{n} 2^{n}} \leq 3-\delta
$$

For the remainder of this section, we shall adopt the above definitions of regular and singular points of a stable minimal hypersurface $M$, and assume the conditions $(\star)$ and $(\star \star)$ on $M$.

The main interior regularity theorem of [Wic-1], which in particular explains rather completely the asymptotic nature of a stable minimal hypersurface satisfying $(\star)$ near any of its branch points with a multiplicity 2 tangent plane, is the following:

Theorem 1.3. For each $\delta \in(0,1)$, there exists a number $\epsilon \in(0,1)$, depending only on $n$ and $\delta$, such that the following is true. If $M$ is an immersed stable minimal hypersurface of $B_{2}^{n+1}(0)$, with $0 \in \bar{M}$ satisfying $(\star)$, ( $\left.\star \star\right)$ and $\int_{M \cap\left(B_{1}(0) \times \mathbf{R}\right)}\left|x^{n+1}\right|^{2} \leq \epsilon$, then $M_{1} \cap\left(B_{1 / 2}(0) \times \mathbf{R}\right)=\operatorname{graph} u$ where $M_{1}$ is the connected component of $\bar{M} \cap\left(B_{1}(0) \times \mathbf{R}\right)$ containing the origin and $u$ is either a single valued or a 2-valued $C^{1, \alpha}$ function on $B_{1 / 2}^{n}(0)$ satisfying

$$
\|u\|_{C^{1, \alpha}\left(B_{1 / 2}(0)\right)} \leq C\left(\int_{M \cap\left(B_{1}(0) \times \mathbf{R}\right)}\left|x^{n+1}\right|^{2}\right)^{1 / 2} .
$$

Here the constants $C \in(0, \infty)$ and $\alpha \in(0,1)$ depend only on $n$ and $\delta$.

Notice that in this theorem, regularity is asserted, of necessity (due to branching), in terms of a multi-valued (2-valued in this case) function $u$. The $C^{1, \alpha}$ regularity of this function amounts geometrically to the following. First, $u$ assigns to each point in its domain a pair of real numbers so that graph $u$ consists of two points above each point of its domain. Furthermore, $u$ has a 2 -valued gradient in the sense that graph $u$ above each point of the domain can be approximated 
by a pair of affine hyperplanes. Finally, the gradient of $u$ (i.e. the unordered pair of gradients of these affine hyperplanes) is uniformly Hölder continuous with respect to the usual metric on the space of unordered pairs of vectors. (Note that this space is not a linear space, eventhough we use the norm $\|\cdot\|$ notation. See [Wic-1] for the precise definition of the $C^{1, \alpha}$ norm of $u$ when $u$ is a 2-valued function.) A simple example of such a function whose graph does not decompose as the union of two intersecting $C^{1, \alpha}$ graphs (as is the case when branch points are present) is $u(z)=\mathcal{R} e z^{3 / 2}$ for $z=x+i y \in B_{1}(0) \subset \mathbf{R}^{2}$. (The graphs of this function is of course not stationary for area. See Section 2 for a construction of stationary (and stable) examples.)

Theorem 1.3 rules out, for instance, the possibility of having a sequence of "necks" connecting two sheets and converging to a branch point of the hypersurface. An example which shows that the theorem fails to hold if we drop the stability assumption (even if we assume embeddedness) is the catenoid. By homothetically scaling down a standard 2-dimensional catenoid in $\mathbf{R}^{3}$, we may make it arbitrarily close to a hyperplane. The neck of the catenoid however persists at all scales.

A description of the proof of Theorem 1.3 will be given at the end of this section.

If we make the stronger hypothesis that $\mathcal{H}^{n-2}(\operatorname{sing} M)=0$, and leave all of the other hypotheses of Theorem 1.3 unchanged, we obtain the stronger conclusion that the 2-valued graph is in fact the union of two separate minimal graphs. This is immediate from Theorem 1.3 in view of the fact that $(i) B_{1 / 2} \backslash \pi(\operatorname{sing} M)$ is simply connected if $\mathcal{H}^{n-2}(\operatorname{sing} M)=0$, where $\pi: \mathbf{R}^{n+1} \rightarrow \mathbf{R}^{n} \times\{0\}$ is the orthogonal projection, and (ii) if $B \subset \mathbf{R}^{n}$ is an open ball, $K \subset B$ is a relatively closed subset with $\mathcal{H}^{n-1}(K)=0$ and if $u: B \backslash K \rightarrow \mathbf{R}$ is a solution of the minimal surface equation, then $u$ extends to a solution $\bar{u}: B \rightarrow \mathbf{R}$ of the minimal surface equation. The latter fact is a theorem of L. Simon [Sim93]. Thus we have

Theorem 1.4. For each $\delta \in(0,1)$, there exists a number $\epsilon \in(0,1)$, depending only on $n$ and $\delta$, such that the following is true. If $M$ is an immersed stable minimal hypersurface of $B_{2}^{n+1}(0)$, satisfying $(\star \star), 0 \in \bar{M}, \mathcal{H}^{n-2}(\operatorname{sing} M)=0$ and $\int_{M \cap\left(B_{1}(0) \times \mathbf{R}\right)}\left|x^{n+1}\right|^{2} \leq \epsilon$, then $M_{1} \cap\left(B_{1 / 2}(0) \times \mathbf{R}\right)=$ graph $u_{0}$ or $M_{1} \cap$ $\left(B_{1 / 2}(0) \times \mathbf{R}\right)=$ graph $u_{1} \cup$ graph $u_{2}$ where $M_{1}$ is the connected component of $\bar{M} \cap\left(B_{1}(0) \times \mathbf{R}\right)$ containing the origin and $u_{i} \in C^{1, \alpha}\left(B_{1 / 2}(0) ; \mathbf{R}\right), i=0,1,2$, satisfy

$$
\left\|u_{i}\right\|_{C^{1, \alpha}\left(B_{1 / 2}(0)\right)} \leq C\left(\int_{M \cap\left(B_{1}(0) \times \mathbf{R}\right)}\left|x^{n+1}\right|^{2}\right)^{1 / 2} .
$$


Here the constants $C \in(0, \infty)$ and $\alpha \in(0,1)$ depend only on $n$ and $\delta$.

In order to estimate, via a dimension reducing argument, the size of the set of non-branch point singularities of a stable minimal hypersurface (see Theorems 1.6 and 1.7 below), it is necessary to understand the nature of such a hypersurface whenever it is weakly close to a union of hyperplanes with multiplicity $\geq 1$. In case the hypersurface satisfies the mass bound $(\star \star)$, this means that we need to understand the behavior of the near either a multiplicity 2 hyperplane or a transversely intersecting pair of (multiplicity 1) hyperplanes. The former case is explained by Theorem 1.3, and the latter by the following theorem ([Wic-2]), which says that whenever a stable minimal hypersurface $M$ satisfying $(\star)$ and $(\star \star)$ is, in $B_{2}^{n+1}(0)$, weakly close to a transversely intersecting pair of hyperplanes $P$ (how close depending on $P$ ), the part of $M$ in a smaller ball consists of the union of two intersecting smooth, minimal graphs over the respective hyperplanes of $P$.

Theorem 1.5. Let $\beta \in(0, \pi / 2]$ and $\delta \in(0,1)$. There exists a number $\epsilon_{1}=$ $\epsilon_{1}(n, \beta, \delta) \in(0,1)$ such that the following holds. If $P=P_{1} \cup P_{2}$ is a union of hyperplanes $P_{1}, P_{2}$ of $\mathbf{R}^{n+1}, \beta \leq \angle\left(P_{1}, P_{2}\right) \leq \pi / 2, M$ is a stable minimal hypersurface of $B_{2}^{n+1}(0)$ with $0 \in \bar{M}$ satisfying $(\star),(\star \star)$ and Hausdorff distance $(M \cap$ $\left.B_{2}^{n+1}(0), P \cap B_{2}^{n+1}(0)\right) \leq \epsilon$, then

$$
\bar{M} \cap B_{1}^{n+1}(0)=\operatorname{graph} u_{1} \cup \operatorname{graph} u_{2}
$$

where $u_{i}: P_{i} \cap B_{1}^{n+1}(0) \rightarrow P_{i}^{\perp}, i=1,2$, are $C^{1, \alpha}$ functions with

$$
\left\|u_{i}\right\|_{C^{1, \alpha}\left(P_{i} \cap B_{1}^{n+1}(0)\right)} \leq C\left(\int_{M \cap B_{2}^{n+1}(0)} \operatorname{dist}^{2}(x, P)\right)^{1 / 2} .
$$

Here $C=C(n) \in(0, \infty), \alpha=\alpha(n) \in(0,1), \angle\left(P_{1}, P_{2}\right)$ denotes the angle between $P_{1}$ and $P_{2}$.

The stability hypothesis in this theorem is used to show that, a priori, the set $\mathcal{S} \subset B_{1}^{n+1}(0) \cap L$ of points $y$ such that $\pi_{L}^{-1}(y) \cap B_{1}^{n+1}(0)$ consists of a pair of embedded, smooth curves has small $(n-1)$-dimensional Hausdorff measure. Here $L$ denotes the axis of $P$, and $\pi_{L}: \mathbf{R}^{n+1} \rightarrow L$ is the orthogonal projection of $\mathbf{R}^{n+1}$ onto $L$. Thus there is, a priori, a "large region" near $L$ in which the hypersurface self-intersects. This fact enables us to use monotonicity-formula-based a priori estimates due to L. Simon ([Sim93]) which show that the height excess of $M$ relative to any nearby pair of hyperplanes does not concentrate near its axis. i.e. the contribution to the height excess in a ball coming from that part of the 
hypersurface which lies in a small tubular neighborhood of the axis is no more than a small multiple of the total height excess in a larger ball. This is central to proving excess improvement - decay of the height excess by a fixed factor at a fixed smaller scale provided the excess at the smaller scale is measured relative to a suitably chosen new pair of hyperplanes - which leads to regularity. Note that the theorem does not hold if we remove the stability hypothesis, as is easily seen by (an appropriately homothetically scaled down version of) Scherk's second minimal surface. This is a smooth connected embedded minimal surface in $\mathbf{R}^{3}$, and by choosing the scaling parameter appropriately, we may make it arbitrarily close to a pair of planes meeting at a right angle. However, near the axis of the intersecting planes, it has many "holes". (See the introduction of [Pit81] for a detailed description of this surface including schematic pictures.)

Combining Theorems 1.4, 1.5, Allard's regularity and integer varifold compactness theorems ([All72], [Sim83]) J. Simons' theorem concerning the non-existence of non-planar stable minimal hypercones of dimension $\leq 6$ ([Sim83]) and the dimension reducing principle of Federer ([Sim83]), we obtained the following compactness theorem. (C.f. Theorem 1.1 above.)

Theorem 1.6. Let $\delta \in(0,1)$. There exists $\sigma=\sigma(n, \delta) \in(0,1 / 2)$ such that the following is true. Suppose $M_{k}$ is a sequence of orientable stable minimal hypersurfaces immersed in $B_{1}^{n+1}(0)$ with $\mathcal{H}^{n-2}\left(\operatorname{sing} M_{k} \cap B_{\sigma}^{n+1}(0)\right)=0$ for each $k$ and $\limsup _{k \rightarrow \infty} \frac{\mathcal{H}^{n}\left(M_{k}\right)}{\omega_{n}} \leq 3-\delta$. Then there exists a stationary varifold $V$ of $B_{1}^{n+1}(0)$ and a closed subset $S$ of spt $\|V\| \cap B_{\sigma}^{n+1}(0)$ with $S=\emptyset$ if $2 \leq n \leq 6$, $S$ discrete if $n=7$ and $\mathcal{H}^{n-7+\gamma}(S)=0$ for every $\gamma>0$ if $n \geq 8$ such that after passing to a subsequence, which we again denote $\{k\}, M_{k} \rightarrow V$ as varifolds and (spt $\|V\| \backslash S) \cap B_{\sigma}^{n+1}(0)$ is an orientable immersed, smooth, stable minimal hypersurface of $B_{\sigma}^{n+1}(0)$.

For the singular set of any weak limit of a sequence of stable minimal hypersurfaces satisfying $(\star)$ and with mass in the unit ball not much greater than twice the mass of the $n$-dimensional Euclidean ball, we have the following decomposition theorem.

Theorem 1.7. There exist numbers $\epsilon=\epsilon(n) \in(0,1)$ and $\sigma=\sigma(n) \in(0,1)$ such that the following is true. If $V$ is the varifold limit of a sequence $\left\{M_{k}\right\}$ of orientable stable minimal hypersurfaces of $B_{1}^{n+1}(0)$ satisfying $0 \in \overline{M_{k}}, \mathcal{H}^{n-2}\left(\operatorname{sing} M_{k} \cap\right.$ $\left.B_{\sigma}^{n+1}(0)\right)<\infty$ and $\frac{\mathcal{H}^{n}\left(M_{k}\right)}{\omega_{n}} \leq 2+\epsilon$, then

$$
\operatorname{sing} V \cap B_{\sigma}^{n+1}(0)=B \cup S
$$


where

(a) $B$ is the set of multiplicity 2 branch point singularities of $V$ in $B_{\sigma}^{n+1}(0)$. Thus, $B$ consists of those points $Z$ of spt $\|V\| \cap B_{\sigma}^{n+1}(0)$ where $V$ has a multiplicity 2 tangent plane but spt $\|V\|$ fails to be immersed at $Z$. Either $B=\emptyset$ or $\mathcal{H}^{n-2}(B)>0$.

(b) $S$ is a relatively closed subset of spt $\|V\| \cap B_{\sigma}^{n+1}(0)$ with $S \cap B=\emptyset$. Furthermore, $S=\emptyset$ if $2 \leq n \leq 6, S$ is a finite set if $n=7$ and $\mathcal{H}^{n-7+\gamma}(S)=0$ for all $\gamma>0$ if $n \geq 8$.

Theorems 1.6 and 1.7 in particular confirm the expectation that a branched stable minimal hypersurface cannot be approximated by a sequence of regular stable, minimal immersions. See [Wic-1] for other consequences of Theorem 1.3, which include a pointwise curvature estimate and a Bernstein type theorem in case $2 \leq n \leq 6$.

In the remainder of this section, we give the main ideas of the proof of Theorem 1.3, essentially as in [Wic-1]. (See section 3 for a simplification.) The main technical ingredient of the proof is a height excess improvement lemma (Lemma 1.8 below) which we now describe. Let $M$ be a stable minimal hypersurface of $B_{2}^{n+1}(0)$ with $0 \in \bar{M}$ satisfying $(\star)$ and $(\star \star), P=P_{1} \cup P_{2}$ an union of affine hyperplanes $P_{1}, P_{2}$ of $\mathbf{R}^{n+1}$, satisfying $P \cap\left(B_{1}(0) \times \mathbf{R}\right) \subset\left\{\left(x^{\prime}, x^{n+1}\right) \in\right.$ $\left.\mathbf{R}^{n+1}:\left|x^{n+1}\right| \leq 1 / 8\right\}$. Here we allow the possibility that $P_{1} \equiv P_{2}$. Define the height excess of $M$ relative to $P$ at scale $\rho \in(0,1]$ by

$$
Q_{M}(\rho, P)=\rho^{-n-2} \int_{M \cap\left(B_{\rho}(0) \times \mathbf{R}\right)} \operatorname{dist}^{2}(x, P)+H_{M}(\rho, P)
$$

where $H_{M}(\rho, P)$ is more or less equal to $\rho^{-n-2} \int_{P \cap\left(B_{\rho}(0) \times \mathbf{R}\right)} \operatorname{dist}^{2}(x, M)$. The precise definition of this term is too technical to describe here, and is not important for describing the main ideas of the lemma and Theorem 1.3. (See [Wic-1], Section 6 for the precise expression.) Indeed, for the proof of Theorem 1.3, we are ultimately only interested in proving decay of the first term of $Q_{M}(\rho, P)$. i.e. an estimate of the form

$$
\rho^{-n-2} \int_{M \cap\left(B_{\rho}(0) \times \mathbf{R}\right)} \operatorname{dist}^{2}(x, P) \leq C \rho^{\nu}
$$

for some fixed $C=C(n, \delta) \in(0, \infty), \nu=\nu(n, \delta) \in(0, \infty)$ and all $\rho \in(0,1 / 8]$, which we establish by proving decay of $Q_{M}(\rho, P)$. The reason why a second term 
like $H_{M}(\rho, P)$ needs to be included in the definition of excess is that we need to make sure that we always pick the "right" to make the excess small relative to. To illustrate this point, consider the case where $M \cap\left(B_{1} \times \mathbf{R}\right)$ consists of two sheets each of which is a graph over $B_{1}(0)$, and suppose that these sheets are lying very close to each other and close to $B_{1}(0)$. Then the quantity $\int_{M \cap\left(B_{1}(0) \times \mathbf{R}\right)} \operatorname{dist}^{2}(x, P)$ will be small if we choose $P$ to be close to the union of the tangent planes to the individual sheets at the points above the origin. It will also be small (of the same order, provided the sheets of $M$ are sufficiently close to each other) if instead we choose one hyperplane of $P$ to be close to a tangent plane, and the other arbitrarily, e.g. at height $1 / 8$ from the origin parallel to $\mathbf{R}^{n} \times\{0\}$. However, the first choice is the "correct" one as far as "finding the tangent planes by iteratively applying an excess improvement lemma" is concerned.

We may now state the excess improvement lemma.

Lemma 1.8. Let $\theta \in(0,1 / 16), \beta \in(0, \theta / 16)$ and $\gamma \in(0, \beta / 16)$. There exist numbers $\epsilon=\epsilon(n, \delta, \theta, \beta, \gamma) \in(0,1 / 2)$ and $\lambda=\lambda(n, \delta) \in(0, \infty)$ such that the following holds. If $M$ is a stable minimal hypersurface of $B_{2}^{n+1}(0)$ with $0 \in \bar{M}$ satisfying $(\star)$ and $(\star \star)$, and $Q_{M}(1, P) \leq \epsilon$ for some pair of affine hyperplanes $P$ with Hausdorff distance $\left(P \cap\left(B_{1}(0) \times \mathbf{R}\right), B_{1}(0)\right) \leq \epsilon$, then there exists a pair of affine hyperplanes $\widetilde{P}$ with Hausdorff distance $\left(P \cap\left(B_{1}(0) \times \mathbf{R}\right), \widetilde{P} \cap\left(B_{1}(0) \times \mathbf{R}\right)\right) \leq$ $C Q_{M}(1, P)$ such that one of the following three options holds:

(A) $Q_{M}(\theta, \widetilde{P}) \leq C_{1} \theta^{\lambda} Q_{M}(1, P)$.

(B) $Q_{M}(\beta, \widetilde{P}) \leq C_{2} \beta^{\lambda} Q_{M}(1, P)$.

(C) $Q_{M}(\gamma, \widetilde{P}) \leq C_{3} \gamma^{\lambda} Q_{M}(1, P)$

Here $C=C(n, \delta, \theta, \beta, \gamma) \in(0, \infty), C_{1}=C_{1}(n, \delta) \in(0, \infty), C_{2}=C_{2}(n, \delta, \theta) \in$ $(0, \infty)$ and $C_{3}=C_{3}(n, \delta, \theta, \beta) \in(0, \infty)$.

Theorem 1.3 is proved by iteratively applying Lemma 1.8 , after choosing $\theta=$ $\theta(n, \delta) \in(0,1 / 16), \beta=\beta(n, \delta) \in(0, \theta / 16)$ and $\gamma=\gamma(n, \delta) \in(0, \beta / 16)$, in that order, so that $C_{1} \theta^{\lambda} \leq 1 / 4, C_{2} \beta^{\lambda} \leq 1 / 4$ and $C_{3} \gamma^{\lambda} \leq 1 / 4$. Specifically, iterating the lemma, with such $\theta, \beta$ and $\gamma$, starting at scale 1 , we obtain, for $j=1,2,3, \ldots$, a sequence of scales $s_{j}$ so that $s_{j}=\theta^{k_{j}} \beta^{l_{j}} \gamma^{m_{j}}$ for some non-negative integers $k_{j}$, $l_{j}, m_{j}$ with $k_{j}+l_{j}+m_{j}=j$ and a sequence of pairs of affine hyperplanes $P_{j}$ with $d_{\mathcal{H}}\left(P_{j} \cap\left(B_{1}(0) \times \mathbf{R}\right), P_{j-1} \cap\left(B_{1}(0) \times \mathbf{R}\right)\right) \leq 4^{-j} Q$ where $Q=Q_{M}\left(1, \mathbf{R}^{n} \times\{0\}\right)$ 
and $Q_{M}\left(s_{j}, P_{j}\right) \leq 4^{-j} Q$. Thus $\left\{P_{j}\right\}$ is a Cauchy sequence and hence converges to a pair of affine hyperplanes $P$. It is standard then, in view of the fact that $\gamma^{j} \leq s_{j} \leq \theta^{j}$, that the estimate (1.1) follows. By changing the base point and repeating the argument, we also conclude that for each $Y \in B_{1 / 2}(0)$, there exists a unique pair of affine hyperplanes $P_{Y}$ such that

$$
\rho^{-n-2} \int_{M \cap\left(B_{\rho}(Y) \times \mathbf{R}\right)} \operatorname{dist}^{2}\left(x, P_{Y}\right) \leq C \rho^{\nu}
$$

for all $\rho \in(0,1 / 8]$. This leads to the conclusions of the theorem. (See [Wic-1], Section 7 for details.)

We conclude this section by briefly describing the proof of Lemma 1.8. Suppose the hypotheses of the lemma are satisfied for some $\epsilon=\epsilon(n, \delta, \theta, \beta, \gamma) \in(0,1 / 2)$ to be chosen. First choose an affine hyperplane $L$ of $\mathbf{R}^{n+1}$ with $L \cap\left(B_{1}(0) \times \mathbf{R}\right) \subset$ $\left\{\left|x^{n+1}\right| \leq 1 / 8\right\}$ so that

$$
\hat{E} \equiv \int_{M \cap\left(B_{1}(0) \times \mathbf{R}\right)} \operatorname{dist}^{2}(x, L) \leq \frac{3}{2} \inf _{L^{\prime}} \int_{M \cap\left(B_{1}(0) \times \mathbf{R}\right)} \operatorname{dist}^{2}\left(x, L^{\prime}\right)
$$

where the inf is taken over all affine hyperplanes $L^{\prime}$ with $L^{\prime} \cap\left(B_{1}(0) \times \mathbf{R}\right) \subset$ $\left\{\left|x^{n+1}\right| \leq 1 / 8\right\}$. Thus, $\hat{E}$ is a "coarse excess" of $M$ measured relative to a single affine hyperplane. Note that since Hausdorff distance $\left(P \cap\left(B_{1}(0) \times \mathbf{R}\right), B_{1}(0)\right) \leq$ $\epsilon$, provided $\epsilon$ is chosen small, $\hat{E}$ will be small. Let $Q=Q_{M}(1, P)$, the "fine excess" of $M$ measured relative a pair of affine hyperplanes. The proof of the lemma is divided into two cases as follows, one of which must hold:

(a) $Q \leq \zeta \hat{E}$

(b) $Q>\zeta \hat{E}$

where $\zeta=\zeta(n, \delta, \theta) \in(0,1 / 2)$ is a fixed number to be chosen sufficiently small. It is convenient to discuss case $(b)$ first.

Case $(b)$ : Take $\zeta=\zeta(n, \delta, \theta)$ to be fixed by the requirements of the analysis in case $(a)$. (See below.) Note first that in case $(b)$, it suffices to prove improvement of $\hat{E}$ at scale $\beta$ or $\gamma$ (aiming for options $(B)$ or $(C)$ of the lemma), for this would then immediately say that $Q$ has improved in view of the defining criterion of case $(b)$. (Note that the constants $C_{2}$ and $C_{3}$ are allowed to depend on $\theta$.) 
To explain the main idea for obtaining excess improvement in this case, first recall that in multiplicity 1 regularity theorems such as Allard's theorem ([All72], [Sim83]) or Schoen-Simon theorem (Theorem 1.2), the excess improvement estimates are obtained by showing that whenever the excess of the minimal submanifold in a cylinder is small, the submanifold in a smaller cylinder can be well approximated by the graph of a harmonic function (or graphs of several separate harmonic functions in the case of Schoen-Simon theorem). What makes this procedure work is the following estimate for harmonic functions: If $v$ is harmonic in $B_{1}(0) \subset \mathbf{R}^{n}$, then for each $\rho \in(0,1 / 2]$,

$$
\rho^{-n-2} \int_{B_{\rho}(0)}(v-l)^{2} \leq C \rho^{2} \int_{B_{1}(0)} v^{2}
$$

where $l: \mathbf{R}^{n} \rightarrow \mathbf{R}$ is the affine function defined by $l(x)=v(0)+D v(0) \cdot x$, and $C=C(n) \in(0, \infty)$ is a fixed constant. (In fact, the pointwise estimate $\sup _{B_{\rho}(0)}|v-l| \leq C \rho^{2}\|v\|_{L^{2}\left(B_{1}(0)\right)}$ holds for $\rho \in(0,1 / 2]$, with $C=C(n)$, in this case, but we only need the weaker version (1.2) for the purposes of minimal surface regularity.) Geometrically, this is an excess improvement estimate for graph $v$, and if we have a good enough approximation of the minimal submanifold by graph $v$ (and we do!), it would yield an excess improvement estimate for the minimal submanifold. (The main point here is that the error terms in the approximation need to be controllable by the excess of the submanifold (in the larger cylinder) itself.)

For the problem at hand, we adopt the same principle and use a type of harmonic approximation, where we show that whenever $\hat{E}$ is small, $M \cap\left(B_{1 / 2}(0) \times \mathbf{R}\right)$ is well approximated by the graph of a "2-valued harmonic" function. F. J. Almgren Jr. used multi-valued harmonic functions in his work on area minimizing currents (of arbitrary dimension and co-dimension), in [Alm83], where harmonic meant Dirichlet energy minimizing. (However, these energy minimizing functions are in general only Hölder continuous, so an estimate like (1.2) is not available for them.) Under our weaker hypothesis of stability, the approximating functions in fact do not satisfy this energy minimizing property, so our two-valued harmonic functions are actually a somewhat different class of functions. Nevertheless, the codimension 1 setting gives them a lot more structure than was shown by Almgren for the energy minimizing functions, and we are able to obtain a sufficiently detailed, asymptotic description of these functions (see Theorem 1.9 below).

What are these two-valued functions? They arise as bow-ups of sequences of stable minimal hypersurface satisfying $(\star)$ and $(\star \star)$ and converging to hyperplanes. Specifically, consider a sequence $\left\{M_{k}\right\}$ of stable minimal hypersurfaces 
satisfying $(\star)$ and $(\star \star)$ with $M_{k}$ in place of $M$ and a fixed $\delta \in(0,1)$, such that $\hat{E}_{k}=\sqrt{\int_{M_{k} \cap\left(B_{1}(0) \times \mathbf{R}\right)} \operatorname{dist}^{2}\left(x, L_{k}\right)} \searrow 0$ for some sequence of affine hyperplanes $L_{k} \rightarrow \mathbf{R}^{n} \times\{0\}$. We generate a (an at most) two-valued function (blow-up) $v$ over $B_{1 / 2}(0)$ by rescaling the height of $M_{k} \cap\left(B_{1}(0) \times \mathbf{R}\right)$ relative to $L_{k}$ by $\hat{E}_{k}$ and passing to the limit (in $W_{l o c}^{1,2}\left(B_{1}(0) ; \mathbf{R}^{2}\right)$ ) as $k \rightarrow \infty$. (An essential step in this process is to show first that given any $\sigma \in(0,1)$, whenever $\hat{E}_{k}$ is sufficiently small, a large part of $M_{k} \cap\left(B_{\sigma}(0) \times \mathbf{R}\right)$ can be expressed as (at most) two graphs over $L_{k}$, and the non-graphical region has $n$-dimensional measure lower order than $\hat{E}_{k}^{2}$. This is done using essentially the same argument as in the corresponding part of [SS81]. The main difference here is that the graphs are no longer disjoint, since $M_{k}$ are not assumed to be embedded.) Notice that $v$ will be single valued unless the mass of $M_{k} \cap\left(B_{1}(0) \times \mathbf{R}\right)$ is sufficiently large. But if $v$ is single valued, then $v$ will be harmonic, and the analysis is trivial in view of the estimate (1.2). So let us consider from now on only those blow-ups that are two-valued. Notice that we may order the two values of $v(x)$ and write $v(x)=\left(v^{+}(x), v^{-}(x)\right)$ with $v^{+}(x) \geq v^{-}(x)$.

Let $\mathcal{F}_{\delta}$ be the class of 2 -valued functions arising as blow-ups, as described in the preceding paragraph, of sequences of stable minimal hypersurfaces satisfying $(\star)$ and $(\star \star)$ and converging to hyperplanes. The members of $\mathcal{F}_{\delta}$ do behave a lot like single-valued harmonic functions, eventhough they are not in general equal to pairs of separate harmonic functions. If $v=\left(v^{+}, v^{-}\right) \in \mathcal{F}_{\delta}$, it is shown in [Wic-1] that graph $v^{+} \cup \operatorname{graph} v^{-}$is, in general, a continuous, branched submanifold of $B_{1}(0) \times \mathbf{R}$ and that $v$ satisfies the estimate given in the following theorem. The subset $S_{v}$ defined in the statement of the theorem is the branching set of $v$. (See Section 3 for an improved version of this theorem.)

Theorem 1.9. Suppose $v=\left(v^{+}, v^{-}\right) \in \mathcal{F}_{\delta}$. Let $S_{v}$ be the (possibly empty) set of points $z \in B_{1 / 2}(0)$ with the property that there exists no $\sigma>0$ such that graph $\left.\left.v^{+}\right|_{B_{\sigma}(z)} \cup \operatorname{graph} v^{-}\right|_{B_{\sigma}(z)}=\operatorname{graph} v^{1} \cup$ graph $v^{2}$ for a pair of harmonic functions $v^{1}, v^{2}: B_{\sigma}(z) \rightarrow \mathbf{R}$. Then, for each $z \in S_{v}$, there exists an affine function $l_{z}: \mathbf{R}^{n} \rightarrow \mathbf{R}$ such that, for all $\rho \in(0,1 / 16)$,

$$
\rho^{-n-2} \int_{B_{\rho}(z)}\left(v^{+}-l_{z}\right)^{2}+\left(v^{-}-l_{z}\right)^{2} \leq C \rho^{\alpha} \int_{B_{1}(0)}|v|^{2}
$$

where $C=C(n, \delta) \in(0, \infty)$ and $\alpha=\alpha(n, \delta) \in(0, \infty)$.

The proof of Theorem 1.9 uses methods and results due to L. Simon [Sim93]; R. Hardt and L. Simon [HS79]; F. J. Almgren Jr. [Alm83] and the author [Wic04]. 
The way we use Theorem 1.9 to obtain excess improvement in the present case (case $(b))$ is as follows. Let $M$ be as in the lemma. By construction of $\mathcal{F}_{\delta}, M$ can be approximated by graph $\left(l+\hat{E} v^{+}\right) \cup \operatorname{graph}\left(l+\hat{E} v^{-}\right)$, where $v=\left(v^{+}, v^{-}\right) \in \mathcal{F}_{\delta}$ and $l: \mathbf{R}^{n} \rightarrow \mathbf{R}$ is the affine function such that graph $l=L$, provided the height excess $\hat{E} \equiv \hat{E}_{M}(1, L)$ of $M$ relative to $L$ is sufficiently small. This can indeed be done in such a way that the error in the approximation is controlled by $\hat{E}$. Now, if $S_{v} \cap B_{\beta}(0)=\emptyset$, then Theorem 1.9 tells us that graph $\left.\left.v^{+}\right|_{B_{\beta}(0)} \cup \operatorname{graph} v^{-}\right|_{B_{\beta}(0)}=\operatorname{graph} v_{1} \cup \operatorname{graph} v_{2}$ for a pair of harmonic functions $v_{1}, v_{2}: B_{\beta}(0) \rightarrow \mathbf{R}$. In this case, taking $\widetilde{P}$ to be the union of the tangent planes to the graphs of $l+\hat{E} v_{1}, l+\hat{E} v_{2}$ at the points $\left(0, l(0)+\hat{E} v_{1}(0)\right)$, $\left(0, l(0)+\hat{E} v_{2}(0)\right)$ respectively, we see, using the estimate (1.2) for single-valued harmonic functions and the defining criterion of case $(b)$ that the conclusions of the lemma hold with option $(C)$. If on the other hand there exists $z \in S_{v} \cap B_{\beta}(0)$, then we take $\widetilde{P}$ to be $\operatorname{graph}\left(l+\hat{E} l_{z}\right)$, where $l_{z}$ is the affine function given by Theorem 1.9, and use the estimate of Theorem 1.9 and the defining property of case $(b)$ to conclude that the lemma holds with option $(B)$.

Case $(a)$ : This says that, provided $\zeta$ is sufficiently small, the fine excess is significantly smaller than the coarse excess. Write $P=P_{1} \cup P_{2}$, where $P_{1}, P_{2}$ are affine hyperplanes. If $P_{1} \cap P_{2} \cap\left(B_{\theta}(0) \times \mathbf{R}\right)=\emptyset$, it can be shown under the hypotheses of case $(a)$ that provided $\zeta$ and $\epsilon$ are sufficiently small, $M \cap\left(B_{\theta / 2}(0) \times \mathbf{R}\right)$ must be embedded. To see this, first note the uniform cone condition for functions $v=\left(v^{+}, v^{-}\right) \in \mathcal{F}_{\delta}$ given in the following lemma. The proof of this lemma uses ideas due to R.Hardt and L. Simon [HS79] and F. J. Almgren Jr. [Alm83].

Lemma 1.10. Let $v=\left(v^{+}, v^{-}\right) \in \mathcal{F}_{\delta}$. Then $v$ is continuous in $B_{1}(0)$. Furthermore, there exists a fixed constant $C=C(n, \delta) \in(0, \infty)$ such that if $z \in$ $B_{1 / 2}(0) \cap\left\{v^{+}(x)=v^{-}(x)\right\}$ then

$$
|v(x)-v(z)| \leq C|x-z|\left(\int_{B_{1}(0)}|v|^{2}\right)^{1 / 2}
$$

for all $x \in B_{1 / 2}(0)$.

Given the lemma, the argument briefly is as follows: If $P_{1} \cap P_{2} \cap\left(B_{\theta}(0) \times \mathbf{R}\right)=\emptyset$, the defining criterion of case $(a)$ says that provided $\zeta=\zeta(n, \delta, \theta)$ is sufficiently small, we may choose an approximating function $v=\left(v^{+}, v^{-}\right) \in \mathcal{F}_{\delta}$ so that $v^{+}, v^{-}$are respectively $L^{2}$ close to two hyperplanes which are apart by a distance bounded above and below by fixed constants depending only on $n$ and $\delta$ 
and which have empty intersection in $B_{\theta}(0) \times \mathbf{R}$. Lemma 1.10 then says that graph $\left.v^{+}\right|_{B_{3 \theta / 4}(0)}$ and graph $\left.v^{-}\right|_{B_{3 \theta / 4}(0)}$ must be disjoint. This in turn implies that $M \cap\left(B_{\theta / 2}(0) \times \mathbf{R}\right)$ must be embedded.

Hence, in case $P_{1} \cap P_{2} \cap\left(B_{\theta}(0) \times \mathbf{R}\right)=\emptyset$, we have by Theorem 1.2 that $M \cap\left(B_{\theta / 4}(0) \times \mathbf{R}\right)$ decomposes as the union of two disjoint graphs $\mathcal{U}_{1}, \mathcal{U}_{2}$ over $B_{\theta / 4}(0)$. Using standard elliptic estimates, it is easy to see in this case that the conclusions of the lemma hold with option $(B)$, provided we take $\widetilde{P}$ to be the union of the two tangent planes to $\mathcal{U}_{1}$ and $\mathcal{U}_{2}$ at the points above the origin.

If on the other hand $P_{1} \cap P_{2} \cap\left(B_{\theta}(0) \times \mathbf{R}\right) \neq \emptyset$, then it can still be shown, based on Lemma 1.10 again, that that part of $M \cap\left(B_{1}(0) \times \mathbf{R}\right)$ which lies outside a small tubular neighborhood of the axis $\Gamma=P_{1} \cap P_{2}$ must be embedded. Moreover, using stability, we can show that near $\Gamma, M$ has "lots" of self-intersections; i.e. lots of points $Z$ with density $\Theta_{M}(Z) \geq 2$. This enables us to use modifications of arguments due to L. Simon ([Sim93]) to show that the fine excess relative to $P$ does not concentrate near $\Gamma$. A blow up argument (blowing up by the fine excess) then implies that $M \cap\left(B_{1 / 2}(0) \times \mathbf{R}\right)$ can be well approximated by the graphs of two harmonic functions over $P_{1}$ and $P_{2}$, which, in view of the standard estimates for harmonic functions (i.e. the estimate (1.2) above), leads to the conclusions of the lemma with option $(A)$, provided we take $\widetilde{P}$ to be the union of the tangent planes to the two approximating harmonic graphs.

\section{Construction of Stable Branched minimal hypersurfaces}

Let $n \geq 2$ be arbitrary. Here we describe a method for constructing a rather rich class of stable branched minimal hypersurfaces of $\mathbf{R}^{n+1}$ of the type considered in the work discussed above. The results described in this section are joint work with L. Simon ([SW]). This construction (see Theorem 2.3) in particular shows that the conclusion of Theorem 1.3 is optimal.

Let $D$ be the open unit disk in $\mathbf{R}^{2}$ centered at the origin, and set $\mathcal{C}=D \times \mathbf{R}^{n-2}$. Consider the degenerate functional

$$
\mathcal{A}_{0}(u)=\int_{\Omega} 4 r^{2} \sqrt{1+\left(4 r^{2}\right)^{-1}\left|D_{x} u\right|^{2}+\left|D_{y} u\right|^{2}} d x d y
$$

where $\Omega$ is a bounded, open subset of $\mathcal{C}, x \in \mathbf{R}^{2}, y \in \mathbf{R}^{n-2}$ and $r=|x|$. Notice that this functional transforms to the non-parametric area functional under the map $T:\left(r e^{i \theta}, y\right) \mapsto\left(r^{2} e^{2 i \theta}, y\right)$. Thus, given bounded continuous boundary data 


$$
\varphi_{0}: \partial D \times \mathbf{R}^{n-2} \rightarrow \mathbf{R}
$$

if we can find a solution $u_{0}$ of the Euler-Lagrange equation of the functional $\mathcal{A}_{0}$ with $u_{0}\left(e^{i \theta}, y\right)=\varphi_{0}\left(e^{i \theta}, y\right)$ for $\left(e^{i \theta}, y\right) \in \partial D \times \mathbf{R}^{n-2}$, and if $u_{0}$ is $C^{1}$ across the axis $r=0$, then the graph $G$ of the 2-valued function

$$
u\left(r e^{i \theta}, y\right)=u_{0}\left(r^{1 / 2} e^{i \theta / 2}, y\right), \quad 0<r \leq 1, \quad 0 \leq \theta \leq 4 \pi, \quad y \in \mathbf{R}^{n-2},
$$

will be a smooth minimal hypersurface with branch point singularities along the $(n-2)$-dimensional $C^{1}$ submanifold $\left\{\left(0, y, u_{0}(0, y)\right): y \in \mathbf{R}^{n-2}\right\}$. (Of course here we also have to assume that $\varphi_{0}\left(e^{i \theta}, y\right) \not \equiv \varphi_{0}\left(-e^{i \theta}, y\right)$ for $\theta \in[0,2 \pi)$ or else $G$ would be a single valued graph taken with multiplicity 2.) So the idea is to establish first the existence of a $C^{2}(\mathcal{C} \backslash\{(x, y): x=0\}) \cap C^{0}(\overline{\mathcal{C}} \backslash\{(x, y): x=0\})$ solution $u_{0}$ to the Euler-Lagrange equation of $\mathcal{A}_{0}$ with arbitrary prescribed bounded continuous boundary data $\varphi_{0}$, and then show that there is a large class of $\varphi_{0}$ with $\varphi_{0}\left(e^{i \theta}, y\right) \not \equiv \varphi_{0}\left(-e^{i \theta}, y\right)$ for which $u_{0}$ must be $C^{1}$ across the singular axis $r=0$.

We proceed as follows. First we show using quasilinear elliptic PDE techniques how to find a stationary point $u_{0}$ of $\mathcal{A}_{0}$ for any given bounded, continuous boundary data. Then we use geometric measure theoretic arguments to analyze, in case $u_{0}$ has a discontinuity at some point $\left(0, y_{0}\right) \in\{0\} \times \mathbf{R}^{n-2}$, the local nature of the graph $G$ of the two valued function $u$ above the point $\left(0, y_{0}\right)$. This will tell us how to chose boundary data so as to eliminate the possibility of getting such a discontinuity. Finally we show that in case $u_{0}$ is continuous across the axis $r=0$, the closure of the graph $G$ of $u$ in $\mathcal{C} \times \mathbf{R}$ must be a $C^{1, \alpha}$ stable, branched minimal hypersurface.

We now proceed to give some details of the above steps. Assume first that $\varphi_{0}: \partial \mathcal{C} \rightarrow \mathbf{R}$ is smooth and periodic in the $y$ variables. Approximate the functional $\mathcal{A}_{0}$ by non-degenerate functionals

$$
\mathcal{A}_{\delta}(u)=\int_{\Omega} 4 r_{\delta}^{2} \sqrt{1+\left(4 r_{\delta}\right)^{-1}\left|D_{x} u\right|^{2}+\left|D_{y} u\right|^{2}} d x d y
$$

where $\delta \in(0,1 / 2)$ and $r_{\delta}$ is any smooth function of $r$ satisfying $\delta / 2 \leq r_{\delta} \leq \delta$ for $0 \leq r<\delta$ and $r_{\delta}=r$ for $r \geq \delta$. Since $\mathcal{A}_{\delta}(u)$ has the form $\int_{\Omega} F_{\delta}(X, u, D u) d x d y$, where $X=(x, y) \in \mathbf{R}^{n}$ and the integrand $F_{\delta}(X, t, p): \mathbf{R}^{n} \times \mathbf{R} \times \mathbf{R}^{n} \rightarrow \mathbf{R}$ is independent of the $t$ variable, any solution $v \in C^{2}(\mathcal{C}) \cap C^{0}(\overline{\mathcal{C}})$ of the Euler-Lagrange equation of $\mathcal{A}_{\delta}$ which is periodic in the $y$ variables satisfies an interior gradient estimate. (See the discussion in $[\mathrm{SW}]$, Section 1.) Standard theory of uniformly 
elliptic quasilinear equations then gives interior estimates for the higher derivatives up to order 2 , and since $u_{\delta}\left(r^{1 / 2} e^{i \theta / 2}, y\right)$ is a solution of the minimal surface equation on any domain of the form $\left\{\left(r e^{\theta}, y\right): r \in(\delta, 1), \theta \in(\alpha, \pi+\alpha)\right\}$, where $\alpha \in[0,2 \pi)$ is arbitrary, we also get derivative estimates at the boundary using barrier constructions for solutions of the minimal surface equation, provided that $v \in C^{2}(\overline{\mathcal{C}})$ and periodic in the $y$ variables, and that $\varphi_{0}$ has bounded derivatives. Thus, by the Leray-Schauder existence theory, we obtain a $C^{2, \alpha}(\overline{\mathcal{C}})$ solution of the Euler-Lagrange equation of $\mathcal{A}_{\delta}$, with boundary values $\varphi_{0}$. By approximation, we may find such a $u_{\delta}$ under the assumption that $\varphi_{0}$ is Lipschitz with respect to the $y$ variables (rather than smooth) with Lipschitz constant independent of the $x$ variables (and $\varphi_{0}$ still periodic in the $y$ variables). Using the derivative estimates, we may then pass to the limit of a subsequence of $u_{\delta_{i}}$, where $\delta_{i} \searrow 0$ is any sequence, to conclude that there exist a $C^{\infty}(\mathcal{C} \backslash\{(x, y): x=0\}) \cap C^{0}(\overline{\mathcal{C}} \backslash\{(x, y): x=0\})$ solution $u_{0}$ of the Euler-Lagrange equation of $\mathcal{A}_{0}$ with boundary data $\varphi_{0}$. Finally, by another approximation argument as described in [SW], Section 1, we may take $\varphi_{0}$ to be merely bounded and continuous rather than Lipschitz and periodic.

Let $u_{0}$ be as above. The following theorem explains the effect on the local structure of $G=\operatorname{graph} u$ of any discontinuity of $u_{0}$ along the axis $\{0\} \times \mathbf{R}^{n-2}$. It says that the part of $\bar{G}$ in some ball centered above an appropriately chosen point close to the discontinuity consists of two smooth, embedded manifolds-withboundary whose common boundary in that ball is a vertical segment.

Theorem 2.1. Suppose the function $u_{0}$ constructed as above has a discontinuity at some point $\left(0, y_{0}\right) \in\{0\} \times \mathbf{R}^{n-2}$ and $\rho_{0} \in(0,1 / 4]$. Then there is a number $\rho_{1} \in\left(0, \rho_{0}\right]$ and a point $\left(0, y_{1}, t_{1}\right) \in B_{\rho_{0}}\left(0, y_{0}\right) \times \mathbf{R}$ such that $B_{\rho_{1}}^{n+1}\left(0, y_{1}, t_{1}\right) \cap$ $\left(\left(0, y_{1}, t_{1}\right)+\{0\} \times \mathbf{R}^{n-2} \times \mathbf{R}\right) \subset \bar{G}, G$ (as an $n$-dimensional integer multiplicity varifold in $\left.\mathbf{R}^{n+1}\right)$ has a unique tangent cone $\mathbf{C}$ at $\left(0, y_{1}, t_{1}\right)$ of the form

$$
\mathbf{C}=\left|\mathbf{H}_{1}\right|+\left|\mathbf{H}_{2}\right|,
$$

where $\mathbf{H}_{1}, \mathbf{H}_{2}$ are distinct $n$-dimensional half-spaces meeting at an angle $\neq \pi$ along the common boundary $\{0\} \times \mathbf{R}^{n-2} \times \mathbf{R},\left|\mathbf{H}_{j}\right|$ is the multiplicity 1 varifold associated with $\mathbf{H}_{j}$ and

$$
\bar{G} \cap B_{\rho_{1}}^{n+1}\left(0, y_{1}, t_{1}\right)=L_{1} \cup L_{2},
$$

where $L_{j}$ is an embedded $C^{\infty}$ manifold-with-boundary, with boundary $\partial L_{j}$ (taken in the open ball $\left.B_{\rho_{1}}^{n+1}\left(0, y_{1}, t_{1}\right)\right)$ given by $\partial L_{j}=B_{\rho_{1}}^{n+1}\left(0, y_{1}, t_{1}\right) \cap\left(\left(0, y_{1}, t_{1}\right)+\right.$ $\left.\mathbf{R}^{n-2} \times \mathbf{R}\right), L_{j}$ has the tangent half-space $\mathbf{H}_{j}$ at the point $\left(0, y_{1}, t_{1}\right)$ and $\left(L_{1} \backslash\right.$ 
$\left.\partial L_{1}\right) \cap\left(L_{2} \backslash \partial L_{2}\right)=\emptyset$.

The proof of this theorem uses Theorem 1.2 , Allard's boundary regularity theorem [All75] and the following removable boundary-discontinuity lemma, whose proof is based on a standard barrier construction. See $[\mathrm{SW}]$ for details.

Lemma 2.2. Let $\Omega \subset \mathbf{R}^{n}$ be open, $x_{0} \in \partial \Omega$ and suppose that $\Omega \cap B_{\rho_{0}}\left(x_{0}\right) \subset U$ for some $\rho_{0}>0$ and open half-space $U$ with $x_{0} \in \partial U$. Let $\varphi: \partial \Omega \cap B_{\rho_{0}}\left(x_{0}\right) \rightarrow \mathbf{R}$ be continuous, and suppose that $u$ is a bounded $C^{2}$ solution of the minimal surface equation in $\Omega$. Suppose also that there exists a compact set $K \subset \partial \Omega$ with $x_{0} \in K$, $\mathcal{H}^{n-1}(K)=0$ such that, for each $y \in \partial \Omega \cap B_{\rho_{0}}\left(x_{0}\right) \backslash K, \lim _{x \rightarrow y, x \in \Omega} u(x)=\varphi(y)$. Then $\lim _{x \rightarrow x_{0}, x \in \Omega} u(x)=\varphi\left(x_{0}\right)$.

Theorem 2.1 immediately tells us that there is a large class of boundary data $\varphi_{0}$ for which $u_{0}$ has to be continuous across $\{0\} \times \mathbf{R}^{n-2}$; namely, those $\varphi_{0}$ which satisfy a $\mathbf{Z}_{k}$ rotational symmetry with respect to the $x$ variables for some odd integer $k \geq 3$, i.e. $\varphi_{0}$ which satisfy, for some odd integer $k \geq 3$, $\varphi_{0}\left(e^{i(\theta+2 \pi / k)}, y\right)=\varphi_{0}\left(e^{i \theta}, y\right)$ for $\theta \in(0,2 \pi]$ and $y \in \mathbf{R}^{n-2}$. For such boundary data, by the maximum principle (which gives uniqueness of solutions to the EulerLagrange equation of $\mathcal{A}_{\delta}$ for given periodic boundary data as considered in the discussion above, implying that $u_{\delta}$ must have the same $\mathbf{Z}_{k}$ symmetry that the boundary data has), we see that $u_{0}$ inherits this symmetry. This means that $G$ must satisfy $S_{k}(G)=G$, where $S_{k}:\left(r e^{i \theta}, y, t\right) \mapsto\left(r e^{i(\theta+2 \pi / k)}, y, t\right)$. Hence, if $u_{0}$ has a discontinuity at some point $\left(0, y_{0}\right) \in\{0\} \times \mathbf{R}^{n-2}$, then the tangent cone $\mathbf{C}$ as in Theorem 2.1 must also satisfy $S_{k}(\mathbf{C})=\mathbf{C}$. But this is impossible since spt $\mathbf{C}$ consists of two distinct half-spaces meeting along their common boundary, and $k$ is odd.

On the other hand, continuity of $u_{0}$ in fact implies that $\mathcal{H}^{n-2}(\bar{G} \cap(\{0\} \times \Omega \times$ $\mathbf{R}))<\infty$ for any bounded subset $\Omega \subset \mathbf{R}^{n-2}$. Using this and the stationarity of $G$, we can modify the standard arguments for single valued solutions of the minimal surface equation to obtain the following existence result for $C^{1, \alpha}$ stable branched minimal immersions. We refer the reader to $[\mathrm{SW}]$ for details.

Theorem 2.3. If the boundary data $\varphi_{0}: \partial D \times \mathbf{R}^{n-2} \rightarrow \mathbf{R}$ is bounded, continuous and has the $\mathbf{Z}_{k}$ symmetry in the $x$ variables for some odd integer $k \geq 3$, and $u_{0}$ is the solution, constructed as above, of the Euler-Lagrange equation of $\mathcal{A}_{0}$ with this boundary data, then the image of the 2-valued mapping 


$$
\Phi:\left(r e^{i \theta}, y\right) \mapsto\left(r e^{i \theta}, y, u_{0}\left(r^{1 / 2} e^{i \theta / 2}, y\right)\right), \quad r \in[0,1], \theta \in[0,4 \pi)
$$

is a $C^{1, \alpha}$ stable branched minimal hypersurface of $\mathcal{C} \times \mathbf{R}$ with boundary values

$$
\Phi\left(e^{i \theta}, y\right)=\left(e^{i \theta}, y, \varphi_{0}\left(r^{1 / 2} e^{i \theta / 2}, y\right)\right), \quad \theta \in[0,4 \pi) .
$$

Finally, we mention that there is also a version of this theorem which gives " $q$ sheeted" branched examples for $q \geq 3$. See [SW].

\section{Asymptotics For the BlOW-UPs of Stable Minimal HyPERSURFACES}

In this section, we make an observation which leads to improvements of Lemmas 1.9 and 1.8. In particular, the discussion here shows that it is not necessary to allow three scales $\theta, \beta$ and $\gamma$ in Lemma 1.8; instead, it is shown here that excess improvement of the hypersurface $M$ under the hypotheses of Lemma 1.8 occurs always at a single fixed smaller scale $\theta$.

We begin by recalling that the class of two-valued harmonic functions considered in [Wic-1] (the class $\mathcal{F}_{\delta}$ discussed in Section 1 above) consists of those functions arising as blow-ups, off affine hyperplanes, of sequences of stable minimal hypersurfaces satisfying $(\star)$ and $(\star \star)$ and weakly converging to a multiplicity 2 affine hyperplane. Here we enlarge this class to the collection of two-valued functions resulting when sequences of stable minimal hypersurfaces, still satisfying $(\star)$ and $(\star \star)$ and weakly converging to a multiplicity 2 affine hyperplane, are blown up by their height excess relative to pairs of affine hyperplanes. We wish to show that the functions in this class satisfy a uniform $L^{2}$ estimate which is the direct analog of the estimate (1.2) for single valued harmonic functions.

In order to define this class precisely and prove the required estimate, let $\delta \in(0,1)$ be fixed and consider a sequence $\left\{M_{k}\right\}$ of immersed stable minimal hypersurfaces of the ball $B_{2}^{n+1}(0)$ satisfying $0 \in \bar{M}_{k}, \mathcal{H}^{n-2}\left(\operatorname{sing} M_{k}\right)<\infty$, $\frac{\mathcal{H}^{n}\left(M_{k} \cap\left(B_{2}^{n+1}(0)\right.\right.}{\omega_{n} 2^{n}} \leq 3-\delta$ and

$$
\int_{M_{k} \cap\left(B_{1}(0) \times \mathbf{R}\right)} \operatorname{dist}^{2}\left(X, P_{k}\right) \searrow 0
$$

for some sequence of pairs of affine hyperplanes $P_{k}$ converging to $\mathbf{R}^{n} \times\{0\}$ (in the sense that $\left.d_{\mathcal{H}}\left(P_{k} \cap\left(B_{1}(0) \times \mathbf{R}\right), B_{1}(0)\right) \rightarrow 0\right)$. For each $k=1,2,3, \ldots$, let $L_{k}$ be any (single) affine hyperplane of $\mathbf{R}^{n+1}$ such that 
Existence and Regularity of Stable Branched Minimal Hypersurfaces 589

$$
\int_{M_{k} \cap\left(B_{1}(0) \times \mathbf{R}\right)} \operatorname{dist}^{2}\left(X, L_{k}\right) \leq \frac{3}{2} \inf _{L} \int_{M_{k} \cap\left(B_{1}(0) \times \mathbf{R}\right)} \operatorname{dist}^{2}(X, L)
$$

where the inf is taken over all affine hyperplanes $L$ of $\mathbf{R}^{n+1}$. Note that we then have $d_{\mathcal{H}}\left(L_{k} \cap\left(B_{1}(0) \times \mathbf{R}\right), B_{1}(0)\right) \rightarrow 0$. Let

$$
\hat{E}_{k}=\sqrt{\int_{M_{k} \cap\left(B_{1}(0) \times \mathbf{R}\right)} \operatorname{dist}^{2}\left(X, L_{k}\right)} .
$$

We consider "blowing up" the hypersurfaces $M_{k}$ by their height excesses $Q_{k} \equiv$ $Q_{M_{k}}\left(1, P_{k}, L_{k}, t_{k}\right)$. Here

$$
\begin{aligned}
& Q_{M}^{2}(\rho, P, L, t) \equiv \\
& \rho^{-n-2} \int_{M \cap\left(B_{\rho}(0) \times \mathbf{R}\right)} \operatorname{dist}^{2}(X, P)+\rho^{-n-2} \int_{P^{\star} \cap\left(\left(B_{\rho / 2}(0) \backslash S_{P}(\rho / 16)\right) \times \mathbf{R}\right)} \operatorname{dist}^{2}\left(X, G_{M}^{(L, t)}(\rho)\right)
\end{aligned}
$$

where the notation is as in Section 6 of [Wic-1]. In order to carry out this blowing up process, we need to consider separately the cases:

(a) $Q_{k} \leq \zeta_{0} \hat{E}_{k}$ for infinitely many $k$;

(b) $Q_{k}>\zeta_{0} \hat{E}_{k}$ for infinitely many $k$.

Here $\zeta_{0}=\zeta_{0}(n, \delta) \in(0,1 / 2)$ is a small constant depending only on $n$ and $\delta$. If we choose $\zeta_{0}$ sufficiently small, then, if case $(a)$ above holds, we see that in view of Lemmas 6.1, 6.2, 5.5, 5.6, and Proposition 3.10 of [Wic-1], the blow-up $v^{ \pm} \in W^{1,2}\left(B_{1 / 2}(0)\right) \cap C^{0}\left(B_{1 / 2}(0)\right)$ of (an appropriately chosen subsequence of) $M_{k}$ by $\hat{E}_{k}$ consists of two harmonic functions; i.e. graph $v^{+} \cup \operatorname{graph} v^{-}=\operatorname{graph} v^{(1)} \cup$ $\operatorname{graph} v^{(2)}$ for a pair of harmonic functions $v^{(1)}, v^{(2)}: B_{1 / 2}(0) \rightarrow \mathbf{R}$. Furthermore, we have that $\left\{x \in B_{1 / 2}(0): v^{(1)}(x)=v^{(2)}(x)\right\} \subset\left\{x: \operatorname{dist}(x, L)<C \zeta_{0}\right\}$ for some affine hyperplane $L$ of $\mathbf{R}^{n} \times\{0\}$ with $L \cap B_{3 / 4}(0) \neq \emptyset$ and $C=C(n) \in(0, \infty)$. This means that we can now blow up the $M_{k}$ 's by their height excesses $Q_{k}$ (note that $Q_{k}$ is of the same order of magnitude as $E_{k} \equiv \sqrt{\int_{M_{k} \cap\left(B_{1}(0) \times \mathbf{R}\right)} \operatorname{dist}^{2}\left(X, P_{k}\right)}$ in this case), either as described in the proof of Lemma 4.1 of [Wic-1] or using Schoen-Simon regularity theorem (Theorem 1.2 above) depending on whether $\left\{x \in B_{1 / 2}(0): v^{(1)}(x)=v^{(2)}(x)\right\}$ is non-empty or empty. This (second) blow up also consists of two harmonic functions $\widetilde{v}^{(1)}, \widetilde{v}^{(2)}: B_{1 / 4}(0) \rightarrow \mathbf{R}$, and hence satisfies the estimate 
$\rho^{-n-2} \int_{B_{\rho}(0)} \operatorname{dist}^{2}\left(\left(x, \widetilde{v}^{+}(x)\right), P_{0}\right)+\operatorname{dist}^{2}\left(\left(x, \widetilde{v}^{-}(x)\right), P_{0}\right) \leq C \rho^{2} \int_{B_{1 / 2}(0)}\left(\widetilde{v}^{+}\right)^{2}+\left(\widetilde{v}^{-}\right)^{2}$

for some pair of affine hyperplanes $P_{0}$ and all $\rho \in(0,1 / 8)$, where $C=C(n) \in$ $(0, \infty)$ and we have used the notation $\widetilde{v}^{+}(x)=\max \left\{\widetilde{v}^{(1)}(x), \widetilde{v}^{(2)}(x)\right\}$ and $\widetilde{v}^{-}(x)=$ $\min \left\{\widetilde{v}^{(1)}(x), \widetilde{v}^{(2)}(x)\right\}$.

In order to prove that a similar estimate holds in case $(b)$, we only need to observe that Theorem 1.9 above (which concerns blow-ups by $\hat{E}_{k}$ ) can be improved to the following result giving a uniform estimate for the functions in $\mathcal{F}_{\delta}$ :

For each $\left(v^{+}, v^{-}\right) \in \mathcal{F}_{\delta}$ we have the estimate

$\rho^{-n-2} \int_{B_{\rho}(0)} \operatorname{dist}^{2}\left(\left(x, v^{+}(x)\right), P\right)+\operatorname{dist}^{2}\left(\left(x, v^{-}(x)\right), P\right) \leq C \rho^{\lambda} \int_{B_{1}(0)}\left(v^{+}\right)^{2}+\left(v^{-}\right)^{2}$

for all $\rho \in(0,1 / 8)$ and some pair of affine hyperplanes $P$, where $C=C(n, \delta) \in$ $(0, \infty)$ and $\lambda=\lambda(n, \delta) \in(0, \infty)$.

For if we have 3.4 for $\left(v^{+}, v^{-}\right) \in \mathcal{F}_{\delta}$, then, it follows directly in view of the defining condition of case $(b)$ that a blow-up $\left(\widetilde{v}^{+}, \widetilde{v}^{-}\right)$of (a subsequence of) $M_{k}$ by $Q_{k}$ (off the corresponding subsequence of $\left\{L_{k}\right\}$ ) must also satisfy 3.4 with $\widetilde{v}^{ \pm}$ in place of $v^{ \pm}$(and a different pair of affine hyperplanes $\widetilde{P}$ in place of $P$ and a different constant $\widetilde{C}=\widetilde{C}(n, \delta)$ in place of $C)$, as required.

In order to establish (3.4) for $\left(v^{+}, v^{-}\right) \in \mathcal{F}_{\delta}$, let $\beta \in(0,1 / 32)$ be arbitrary for the moment, and consider a function $\left(v^{+}, v^{-}\right) \in \mathcal{F}_{\delta}$. If there is a point $z \in S_{v} \cap B_{\beta}(0)$ (notation as in Theorem 1.9), then by Theorem 1.9, we have that $\beta^{-n-2} \int_{B_{\beta}(0)}\left(v^{+}-l_{z}\right)^{2}+\left(v^{-}-l_{z}\right)^{2} \leq(1+|z| / \beta)^{n+2}(\beta+|z|)^{-n-2} \int_{B_{\beta+|z|}(z)}\left(v^{+}-\right.$ $\left.l_{z}\right)^{2}+\left(v^{-}-l_{z}\right)^{2} \leq C_{1} \beta^{\lambda} \int_{B_{1}(0)}\left(v^{+}\right)^{2}+\left(v^{-}\right)^{2}$ where $C_{1}=C_{1}(n, \delta)$ and $\lambda=\lambda(n, \delta)$. If on the other hand $S_{v} \cap B_{\beta}(0)=\emptyset$, then graph $\left.\left.v^{+}\right|_{B_{\beta}(0)} \cup \operatorname{graph} v^{-}\right|_{B_{\beta}(0)}=$ graph $v_{1} \cup$ graph $v_{2}$ for a pair of harmonic functions $v_{1}, v_{2}: B_{\beta}(0) \rightarrow \mathbf{R}$, so that in this case, we have that $(\sigma \beta)^{-n-2} \int_{B_{\sigma \beta}(0)} \operatorname{dist}^{2}\left(\left(x, v^{+}(x)\right), P\right)+\operatorname{dist}^{2}\left(\left(x, v^{-}(x)\right), P\right)$ $\leq C_{2} \beta^{-n-2} \sigma^{2} \int_{B_{\beta}(0)}\left(v^{+}\right)^{2}+\left(v^{-}\right)^{2}$ for some pair of affine hyperplanes $P$, where $C_{2}=C_{2}(n)$. Choosing $\beta=\beta(n, \delta) \in(0,1 / 32)$ so that $C_{1} \beta^{\lambda} \leq 1 / 4$ we thus have 
the following:

There exists a constant $\beta=\beta(n, \delta) \in(0,1 / 32)$ such that if $\left(v^{+}, v^{-}\right) \in \mathcal{F}_{\delta}$, then either

$$
\beta^{-n-2} \int_{B_{\beta}(0)}\left(v^{+}-l\right)^{+}+\left(v^{-}-l\right)^{2} \leq \frac{1}{4} \int_{B_{1}(0)}\left(v^{+}\right)^{2}+\left(v^{-}\right)^{2}
$$

for some affine function l, or

$$
\begin{gathered}
(\sigma \beta)^{-n-2} \int_{B_{\sigma \beta}(0)} \operatorname{dist}^{2}\left(\left(x, v^{+}(x)\right), P\right)+\operatorname{dist}^{2}\left(\left(x, v^{-}(x)\right), P\right) \leq \\
C_{2} \beta^{-n-2} \sigma^{2} \int_{B_{\beta}(0)}\left(v^{+}\right)^{2}+\left(v^{-}\right)^{2}
\end{gathered}
$$

for some pair of affine hyperplanes $P$ and all $\sigma \in(0,1 / 2)$. Here $C_{2}=C_{2}(n)$.

In view of the fact that for any $\rho \in(0,1)$ and any affine function $l$, $\frac{\left(v_{\rho}^{+}-l, v_{\rho}^{-}-l\right)}{\left(\rho^{-n-2} \int_{B_{\rho}(0)}\left(v^{+}-l\right)^{2}+\left(v^{-}-l\right)^{2}\right)^{1 / 2}} \in \mathcal{F}_{\delta}$ whenever $\left(v^{+}, v^{-}\right) \in \mathcal{F}_{\delta}$, where $v_{\rho}^{ \pm}(x)=$ $\frac{1}{\rho / 2} v^{ \pm}\left(\frac{1}{2} \rho x\right)$, we may apply the above result iteratively to conclude that:

(i) either there exists an infinite sequence of affine functions $l_{j}, j=0,1,2, \ldots$, with $l_{0} \equiv 0$, such that

$$
\begin{aligned}
\left(\beta^{j}\right)^{-n-2} \int_{B_{\beta^{j}}(0)}\left(v^{+}-l_{j}\right)^{2} & +\left(v^{-}-l_{j}\right)^{2} \\
& \leq 4^{-1}\left(\beta^{j-1}\right)^{-n-2} \int_{B_{\beta^{j-1}}(0)}\left(v^{+}-l_{j-1}\right)^{2}+\left(v^{-}-l_{j-1}\right)^{2} \\
& \leq 4^{-j} \int_{B_{1}(0)}\left(v^{+}\right)^{2}+\left(v^{-}\right)^{2}
\end{aligned}
$$

for each $j=1,2,3, \ldots$ or

(ii) there exist an integer $j^{\star} \geq 0$, affine functions $l_{0}, l_{1}, \ldots, l_{j^{\star}}$ with $l_{0} \equiv 0$ and a pair of affine hyperplanes $P$ such that 


$$
\begin{array}{cc}
\left(\sigma \beta^{j^{\star}+1}\right)^{-n-2} \int_{B_{\sigma j^{\star}+1}(0)} & \operatorname{dist}^{2}\left(\left(x, v^{+}(x)\right), P\right)+\operatorname{dist}^{2}\left(\left(x, v^{-}(x)\right), P\right) \\
\leq C_{2} \sigma^{2}\left(\beta^{j^{\star}+1}\right)^{-n-2} \int_{B_{\beta j^{\star}+1}(0)}\left(v^{+}-l_{j^{\star}}\right)^{2}+\left(v^{-}-l_{j^{\star}}\right)^{2} \\
\leq C_{2} \beta^{-n-2} \sigma^{2}\left(\beta^{j^{\star}}\right)^{-n-2} \int_{B_{\beta^{\star}}(0)}\left(v^{+}-l_{j^{\star}}\right)^{2}+\left(v^{-}-l_{j^{\star}}\right)^{2} \\
\leq C_{2} \beta^{-n-2} \sigma^{2} 4^{-j^{\star}} \int_{B_{1}(0)}\left(v^{+}\right)^{2}+\left(v^{-}\right)^{2}
\end{array}
$$

for all $\sigma \in(0,1 / 2)$ and, in case $j^{\star} \geq 1$,

$$
\begin{aligned}
\left(\beta^{j}\right)^{-n-2} \int_{B_{\beta^{j}}(0)}\left(v^{+}-l_{j}\right)^{2} & +\left(v^{-}-l_{j}\right)^{2} \\
& \leq 4^{-1}\left(\beta^{j-1}\right)^{-n-2} \int_{B_{\beta^{j-1}}(0)}\left(v^{+}-l_{j-1}\right)^{2}+\left(v^{-}-l_{j-1}\right)^{2} \\
& \leq 4^{-j} \int_{B_{1}(0)}\left(v^{+}\right)^{2}+\left(v^{-}\right)^{2}
\end{aligned}
$$

for $j=1,2, \ldots, j^{\star}$.

If $(i)$ holds, it is standard that

$$
\rho^{-n-2} \int_{B_{\rho}(0)}\left(v^{+}-l\right)^{2}+\left(v^{-}-l\right)^{2} \leq C \rho^{\lambda} \int_{B_{1}(0)}\left(v^{+}\right)^{2}+\left(v^{-}\right)^{2}
$$

for some affine hyperplane $l$ and all $\rho \in(0,1 / 4]$, where $C=C(n, \delta)$ and $\lambda=$ $\lambda(n, \delta)$. If on the other hand (ii) holds, it is again not difficult to see first that, in case $j^{\star} \geq 1$,

$$
\left(\beta^{j}\right)^{-n-2} \int_{B_{\beta^{j}}(0)} \operatorname{dist}^{2}\left(\left(x, v^{+}(x)\right), P\right)+\operatorname{dist}^{2}\left(\left(x, v^{-}(x)\right), P\right) \leq C 4^{-j} \int_{B_{1}(0)}\left(v^{+}\right)^{2}+\left(v^{-}\right)^{2}
$$

for each $j=1,2, \ldots, j^{\star}$ and hence that

$\rho^{-n-2} \int_{B_{\rho}(0)} \operatorname{dist}^{2}\left(\left(x, v^{+}(x)\right), P\right)+\operatorname{dist}^{2}\left(\left(x, v^{-}(x)\right), P\right) \leq C \rho^{\lambda} \int_{B_{1}(0)}\left(v^{+}\right)^{2}+\left(v^{-}\right)^{2}$

for all $\rho \in(0,1 / 4]$, where $C=C(n, \delta)$ and $\lambda=\lambda(n, \delta)$. (To see this last inequality, given $\rho \in(0,1 / 4]$, consider the cases $\beta^{j+1} \leq 2 \rho<\beta^{j}$ for some $j \in\left\{0,1,2, \ldots, j^{\star}\right\}$ 
or $2 \rho<\beta^{j^{\star}+1}$. If the former holds and $j^{\star} \geq 1$, use (3.6); else use (3.5) noting that $\rho=\sigma \beta^{j^{*}+1}$ for some $\sigma \in(0,1 / 2)$ and $\beta<1 / 2$.) Thus in either of the cases (i) and (ii), we have the desired estimate (3.4).

Remark: Note that the estimate (3.4) implies that if $v=\left(v^{+}, v^{-}\right) \in \mathcal{F}_{\delta}$, then for each $z \in B_{1 / 4}(0)$ there exitst a pair of affine hyperplanes $P_{z}$ such that

$\rho^{-n-2} \int_{B_{\rho}(z)} \operatorname{dist}^{2}\left(\left(x, v^{+}(x)\right), P_{z}\right)+\operatorname{dist}^{2}\left(\left(x, v^{-}(x)\right), P_{z}\right) \leq C \rho^{\lambda} \int_{B_{1}(0)}\left(v^{+}\right)^{2}+\left(v^{-}\right)^{2}$

for all $\rho \in(0,1 / 32)$, where $C=C(n, \delta) \in(0, \infty)$ and $\lambda=\lambda(n, \delta) \in(0, \infty)$. This in turn implies that $v$ is a 2-valued $C^{1, \alpha}$ function (with $\alpha=\lambda / 2$ ) in $B_{1 / 4}(0)$ (in the sense defined in [Wic-1], Section 2) with $\|v\|_{C^{1, \alpha}\left(B_{1 / 4}(0)\right)} \leq C\|v\|_{L^{2}\left(B_{1}(0)\right)}$, where $C=C(n, \delta)$.

As a direct consequence of the preceding discussion, we have the following height excess improvement lemma, which is a simpler version of Lemma 1.8 above.

Lemma 3.1. Let $\delta \in(0,1)$ and $\theta \in(0,1 / 16)$. There exists a number $\epsilon=$ $\epsilon(n, \delta, \theta) \in(0,1 / 2)$ such that the following holds. Let $M$ be a stable minimal hypersurface of $B_{2}^{n+1}(0)$ with $0 \in \bar{M}$ satisfying $(\star),(\star \star)$ and $Q_{M}(1, P, L, t) \leq \epsilon$ for some pair of affine hyperplanes $P$ with Hausdorff distance $\left(P \cap\left(B_{1}(0) \times\right.\right.$ $\left.\mathbf{R}), B_{1}(0)\right) \leq \epsilon$, an affine hyperplane $L \in \mathcal{A}(M, 1)$ and a number $t \in \mathcal{R}(M, L, 1)$, where $\mathcal{A}(M, \rho)$ and $\mathcal{R}(M, L, \rho)$ are as defined in [Wic-1], Section 6. Then there exists a pair of affine hyperplanes $\widetilde{P}$ with Hausdorff distance $\left(P \cap\left(B_{1}(0) \times \mathbf{R}\right), \widetilde{P} \cap\right.$ $\left.\left(B_{1}(0) \times \mathbf{R}\right)\right) \leq C Q_{M}(1, P, L, t)$, an affine hyperplane $\widetilde{L} \in \mathcal{A}(M, \theta)$ and a number $\widetilde{t} \in \mathcal{R}(M, \widetilde{L}, \theta)$ such that

$$
Q_{M}(\theta, \widetilde{P}, \widetilde{L}, \widetilde{t}) \leq C_{1} \theta^{\lambda} Q_{M}(1, P, L, t) .
$$

Here $C=C(n, \delta, \theta) \in(0, \infty), C_{1}=C_{1}(n, \delta) \in(0, \infty)$ and $\lambda=\lambda(n, \delta) \in(0, \infty)$.

\section{REFERENCES}

[All72] W. K. Allard. On the first variation of a varifold. Ann. of Math. 95 (1972), 417-491.

[All75] W. K. Allard. On the first variation of a varifold-boundary behavior. Ann. of Math. 101 (1975), 418-446.

[Alm83] F. J. Almgren Jr. Almgren's big regularity paper: Q-valued functions minimizing Dirichlet's integral and the regularity of area minimizing rectifiable currents up to codimension 2. World Scientific (2000). 
[HS79] R. Hardt, L. Simon. Boundary regularity and embedded solutions for the oriented Plateau problem. Ann. of Math. 110 (1979), 436-486.

[Ilm96] T. Ilmanen. A strong maximum principle for singular minimal hypersurfaces. Calc. Var. 4 (1996), 443-467.

[Pit81] J. Pitts. Existence and regularity of minimal surfaces on Riemannian manifolds. Princeton University press (1981).

[SS81] R. Schoen, L. Simon. Regularity of stable minimal hypersurfaces. Comm. Pure and Appl. Math. 34 (1981), 742-797.

[Sim83] L. Simon. Lectures on geometric measure theory. Proc. Centre Math. Anal., Austral. Nat. Univ., Canberra 3 (1983).

[Sim93] L. Simon. Cylindrical tangent cones and the singular set of minimal submanifolds. Journal of Differential Geometry 38 (1993), 585-652.

[Sim93] L. Simon. On a theorem of De Giorgi and Stampacchia. Math. Zeit. 155 (1977), 199204.

[SW] L. Simon, N. Wickramasekera. Stable branched minimal immersions with prescribed boundary. Journal of Differential Geometry 75 (2007), 143-173.

[Wic04] N. Wickramasekera. A rigidity theorem for stable minimal hypercones. Journal of Differential Geometry 68, (2004), 433-514.

[Wic-1] N. Wickramasekera. A regularity and compactness theory for immersed stable minimal hypersurfaces of multiplicity at most 2. Preprint.

[Wic-2] N. Wickramasekera. Some multiplicity 1 regularity theorems for minimal submanifolds. Preprint.

Neshan Wickramasekera

University of California, San Diego

9500 Gilman Drive, La Jolla, CA 92093, USA

E-mail: nwickram@math.ucsd.edu 